\title{
Influence de la vitesse et de la direction du vent sur la capture des pucerons ailés par un piège à fils englués
}

Gérard LABONNE, Jean-Bernard QUIOT (*)

I.N.R.A., Laboratoire de la Chaire d'Ecologie animale et de Zoologie agricole. E.N.S.A.M., F 34060 Montpellier Cedex.

$\left(^{*}\right)$ L.P.R.C., I.N.R.A.-Virologie. C.I.R.A.D., B.P. 5035 , F 34032 Montpellier.

L'influence de la vitesse du vent sur l'efficacité de capture d'un piège à fils englués a été étudiée à l'aide de pièges placés en tandem sur un support à girouette. Les captures de pucerons lâchés au vent des pièges permettent de mettre en évidence une bonne corrélation linéaire négative $(R=-0,94)$ entre les proportions de pucerons capturés et les vitesses de vent.

L'influence de la direction du vent a été étudiée de façon indirecte en comparant les captures réalisées par des pièges ayant des écartements de fils différents. On en déduit que les proportions relatives des différentes espèces capturées ne sont pas modifiées par les variations de direction du vent, et que le nombre de pucerons capturés subit une modification négligeable jusqu'à un angle d'incidence de $43^{\circ}$, au moins, du vent par rapport au plan du piège. Des modalités pratiques d'utilisation de ces pièges en sont déduites.

Mots clés additionnels : Epidémiologie, virus non-persistant.

The influence of wind speed on the trapping efficiency of a sticky fishing-line trap has been studied by the mean of double traps fixed on a rotating stand directed by a wind vane. Analysis of the catches of aphids released to windward of the traps showed a good linear negative correlation $(R=-0.94)$ between the proportions of trapped aphids and the speed of the wind. The influence of the direction of the wind has been studied indirectly using traps with different spacing between the sticky threads. It is deduced that the relative proportions of the different species did not change, and that the number of trapped aphids is likely the same up to an angle of $43^{\circ}$, at least, of the wind relative to the plane of the trap. Some practical considerations about the use of the sticky fishing-line traps are deduced from these results.

Additional key words : Epidemiology, non-persistent aphid-borne viruses.

\section{INTRODUCTION}

L'étude de l'épidémiologie des viroses transmises par pucerons sur le mode non-persistant réclame, entre autres, l'utilisation d'un piège adapté permettant de mesurer l'évolution des populations de pucerons ailés à l'intérieur d'un champ.

Pour fournir des données utilisables, un tel piège doit être doté des qualités suivantes: sensibilité, fiabilité, simplicité d'emploi, et assurer un piégeage non sélectif des différentes espèces de pucerons présentes dans la parcelle.

Depuis 1983, nous avons été amenés à proposer un modèle de piège adapté à cette tâche (LABONNE et al., 1983). Basé sur le principe d'un piège d'interception, il est constitué d'une nappe de fils de polyamide transparents englués tendus sur un cadre et il est placé verticalement sur un support. Les fils sont espacés régulièrement et constituent un filtre sur lequel les insectes en vol viennent se coller. Des essais précédents (LABONNE et al., 1983, 1988a) ont montré que ce piège, de réalisation peu coûteuse et assez simple d'emploi, était fiable, répétitif, plus sensible que le piège jaune à eau et que, comme les pièges à succion, il fournissait des captures non sélectives de l'aphidofaune ailée.

Dans cette note, nous présentons les résultats d'expériences complémentaires qui ont été réalisés pour mesurer l'influence de la vitesse et de la direction du vent sur la proportion de pucerons qui restent retenus par le piège par rapport à ceux qui le traversent. 


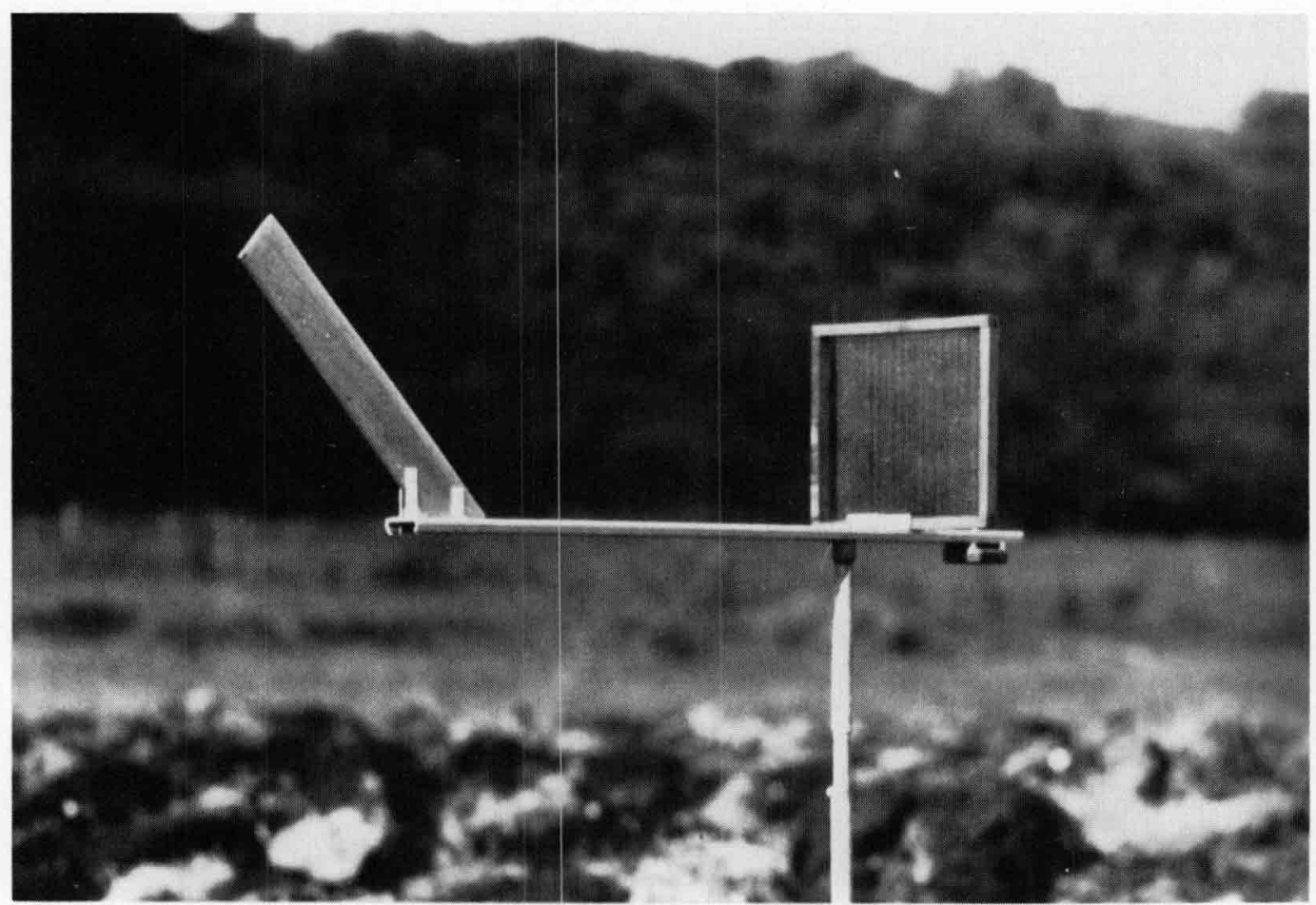

Figure 1

Piege a fils englues place sur son support à giroucthe.

Sticky thread trap on its support with wind-1'anc.

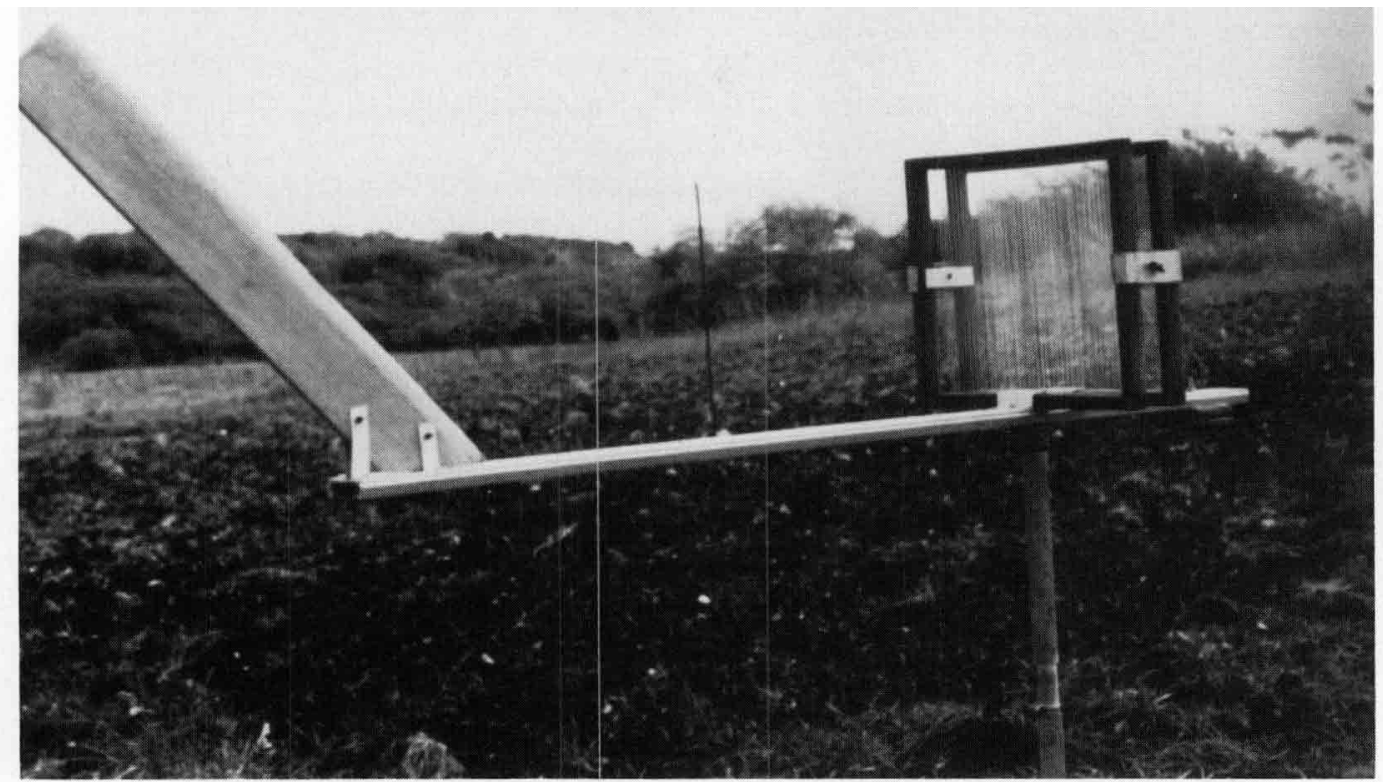

Fïgure 2

Montage en tandem de 2 pièges à fits englués sur leur support à girouette.

Pair of sticky thread traps on support. 


\section{MATÉRIEL ET MÉTHODES}

Les essais sont réalisés en conditions naturelles sous climat tropical, en Guadeloupe. Le vent assez constant (alizé) est mesuré en vitesse et direction avec une anémogirouette Lambrecht. Les pièges sont placés sur des bâtis mobiles à $1,50 \mathrm{~m}$ du sol, à la même hauteur que le capteur de lanémogirouette. Ils sont munis d'une girouette qui leur permet de se maintenir face au vent (figs. 1 et 2).

\section{A. Mesure de l'influence de la vitesse du vent}

Pour mesurer l'influence de la vitesse du vent sur la proportion de pucerons retenus par le piège, on place sur le bâti mobile 2 pièges en tandem espacés l'un de l'autre de $5 \mathrm{~cm}$ (fig. 2). Les pucerons capturés proviennent soit de la population naturelle d'ailés en vol dans la zone, soit d'ailés d'Aphis gossypii mis dans un réceptacle placé sur le bâti mobile à $40 \mathrm{~cm}$ au vent des pièges. A partir des nombres $\mathrm{C} 1$ et $\mathrm{C} 2$ de pucerons capturés par les pièges avant et arrière au cours d'une expérience, il est possible de calculer la proportion $P$ de pucerons retenus par un piège :

$$
\mathrm{P}=(\mathrm{Cl}-\mathrm{C} 2) / \mathrm{C} 1,
$$

tandis que le nombre réel $\mathrm{N}$ de pucerons ayant traversé le piège avant (retenus ou non) est donné par :

$$
\mathrm{N}=\mathrm{C} 1 / \mathrm{P} \text {. }
$$

\section{B. Effet sur les captures de la direction du vent par rapport au plan du piège}

Cet effet peut s'interpréter géométriquement et résulte de 2 causes qui se contrecarrent en partie. Lorsque le vent cesse d'avoir une incidence normale au plan du piège, la surface de capture diminue comme le sinus de l'angle que fait le vent avec le plan de piégeage, ce qui réduit le nombre de pucerons pouvant être interceptés. Mais, dans le même temps, l'intervalle entre les fils subit une réduction apparente proportionnelle aussi au sinus du même angle, réduction qui peut contribuer à assurer un meilleur filtrage de l'air et donc un taux de capture accru.

Les mesures d'incidence du vent sur le plan du piège sont difficiles à réaliser en conditions naturelles. On a donc été amené à les remplacer par des expériences faisant intervenir des pièges présentant des écartements de fils différents : un modèle standard d'une surface de $25 \times 25 \mathrm{~cm}$ avec un écartement entre les fils de $3,1 \mathrm{~mm}$ et un modèle à maille fine de $25 \times 25 \mathrm{~cm}$ avec un écartement de $2,1 \mathrm{~mm}$ entre les fils. Ces 2 modèles sont montés sur des supports à girouette les maintenant face au vent dominant (fig. 1). Le calcul montre que les intervalles de $2,1 \mathrm{~mm}$ entre les fils correspondent aux intervalles entre les fils du piège standard qui recevrait le vent sous une incidence de $43^{\circ}$ par rapport au plan du piège.

\section{RÉSULTATS}

\section{A. Influence de la vitesse du vent sur la proportion de pucerons retenus par un piège à fils englués}

On a réalisé 25 lâchers de pucerons ailés au vent des pièges placés en tandem. Chaque expérience a duré
1 heure. Les vitesses de vent ont varié de $0,87 \mathrm{~m} / \mathrm{s}$ à $3,41 \mathrm{~m} / \mathrm{s}$ d'une expérience à l'autre et ont pu être regroupées en 7 classes. Un total de 689 pucerons a été capturé par les pièges.

On constate (fig. 3) qu'il apparaît une bonne corrélation linéaire négative $(R=-0,94)$ entre les vitesses moyennes de vent mesurées pendant les expériences et la proportion de pucerons retenus sur le piège au vent. L'équation de la droite de régression calculée selon la méthode des moindres carrés est :

$$
\mathrm{P}=0,91-0,13 \mathrm{~V}
$$

$\mathrm{V}$ étant la vitesse de vent exprimée en $\mathrm{m} / \mathrm{s}$.

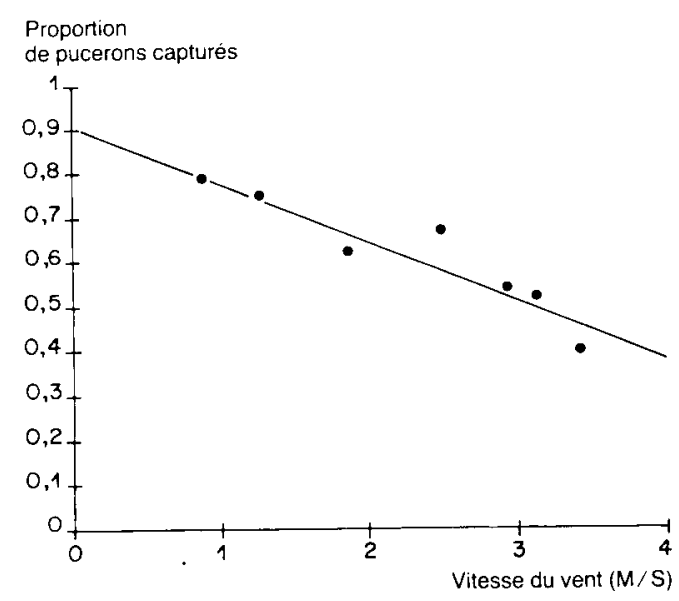

Figure 3

Relation entre la vitesse du vent et la proportion de pucerons capturés par un piège à fils englués. Droite de régression : $P=0,91-0,13 \mathrm{~V}$, coefficient de corrélation linéaire : $R=0,94$

Relation between wind speed and the proportion of aphids trapped by a sticky thread trap. Regression line : $P=0.91-0.13 \mathrm{~V} ;$ Pearson correlation coefficient : $R=-0.94$ )

\section{B. Influence de la direction du vent sur l'efficacité du piège}

Les pièges à maille standard et à maille fine ont été utilisés sur support à girouette pour capturer en conditions naturelles les pucerons ailés au cours de 2 périodes de 154 et 147 jours pendant les saisons sèches 1981-1982 et 1982-1983, les pièges étant relevés 2 fois par semaine. La vitesse moyenne du vent pendant le jour (période de vol des pucerons) a été de $2,5 \mathrm{~m} / \mathrm{s}$ à la hauteur des pièges.

Le dénombrement et l'identification des captures (tabl. 1) font apparaître que les structures des populations de pucerons piégées par les 2 modèles de pièges sont très proches l'une de l'autre. On peut en déduire que, dans les limites de l'expérimentation réalisée, le resserrement de la maille des fils ne modifie pas la composition de l'aphidofaune capturée. On peut en induire que les variations d'angle d'incidence du vent par rapport au plan des fils englués, bien qu'entraînant une réduction apparente des intervalles, ne devrait pas entraîner une modification de la composition de l'aphidofaune capturée, au moins tant que la distance entre fils n'est pas inférieures à $2,1 \mathrm{~mm}$ et pour des espèces de taille analogue à celle des espèces capturées dans cet essai. 
TABLEAU I

Comparaison des effectifs par espèces de pucerons capturés par un piège à maille standard at un piège à maille fine. Comparison of the numbers of aphids which were caught by a trap with standard or fine mesh for each species.

\begin{tabular}{|c|c|c|c|c|}
\hline \multirow{2}{*}{ Période } & \multicolumn{2}{|c|}{ Saison $81-82$} & \multicolumn{2}{|c|}{ Saison 82-83 } \\
\hline & Standard & Fine & Standard & Fine \\
\hline \multicolumn{5}{|l|}{ Espèce : } \\
\hline Anocia sp. & () & 1 & 0 & 1 \\
\hline Aphis cirricola van der Goot & 1 & 0 & 0 & 0 \\
\hline Aphis craccivora Koch & 31 & 47 & 82 & 108 \\
\hline Aphis gossypii Glover & 27 & 30 & 37 & 56 \\
\hline Aphis nerii Boyer de Fonscolombe & 1 & 1 & 4 & 10 \\
\hline Carolinaia sp. & 0 & l & 0 & 3 \\
\hline Hysteroneura setariae Thomas & () & 3 & 0 & 1 \\
\hline Lipaphis ervsimi Kaltenbach & 1 & 1 & 9 & 14 \\
\hline Melanaphis sacchari Zechntner & 0 & 0 & 2 & 0 \\
\hline Rhopalosiphum maidis Fitch & 1 & 2 & 6 & 13 \\
\hline Rhopalosiphum rufiabdominalis Sasaki & 2 & 2 & 2 & 3 \\
\hline Sipha flava Forbes & 15 & 20 & 71 & 92 \\
\hline Tetraneura nigriabdominalis Sasaki & 8 & 12 & 2 & 4 \\
\hline Autres & 0 & 3 & 1 & 0 \\
\hline Total & 88 & 124 & 216 & 305 \\
\hline
\end{tabular}

La comparaison des nombres de pucerons capturés fait apparaître que, à surface d'interception égale, le piège à maille fine capture 40 p. 100 d'individus de plus que le piège à maille standard. Toutefois, lorsqu'on ramène la surface piégeante du piège à maille fine à ce:lle qu'aurait eu un piège standard recevant le vent sous une incidence de $43^{\circ}$ (valeur correspondant au sinus du rapport des 2 écartements), on constate que le nombre de pucerons capturés ne représente que 96 p. 100 des captures réalisées lorsque le vent est normal au plan de piégeage (tabl. 2).

\section{TABLEAU 2}

Comparaison des effectifs de puccrons capturés par un piège à maille standard $(S)$ et un piège à maille fine dont la surface piégeante est corrigée de facon à représenter celle d'un piège à maille standard recevant le vent sous un angle dincidence de $43^{\circ}$ ( $F$ corrigé) .

Comparison of the numbers of aphids which were caugh by a trap nith standard mesh (S) and by a trap with fine mesh for which the trapping area has been corrected in order to represent the same area than a standard trap recieiving the wind at a $43^{\circ}$ angle of incidence ( $F$ corrige)

\begin{tabular}{lcc}
\hline \hline Période & Saison $81-82$ & Saison $82-83$ \\
\hline S : piège à maille standard & 88 & 216 \\
\hline F : piège à maille fine & 124 & 305 \\
\hline F corrigé & 84 & 207 \\
\hline Rapport F corrigé/S & 0,96 & 0.96 \\
\hline \hline
\end{tabular}

On peut en déduire que la modification de l'angle d'incidence du vent par rapport au plan du piège, au moins jusqu'à un angle de $43^{\circ}$, ne modifie que très peu l'efficacité du piège.

\section{DISCUSSION ET CONCLUSION}

Le fait que l'efficacité de ce type de piège diminue en fonction de la vitesse du vent incident constitue un défaut qui en limite les possibilités d'utilisation. Toutefois, la mise en évidence d'une relation linéaire entre efficacité et vitesse du vent montre que, au moins pour les vitesses de vent inférieures à $4 \mathrm{~m} / \mathrm{s}$, ce type de défaut peut être en partie corrigé en associant au piégeage une mesure anémométrique. Il faut aussi noter que l'on a peu de données sur la sensibilité au vent des autres modes de piégeage des pucerons couramment utilisés en épidémiologie, en dehors de certains pièges à succion (JOHNSON, 1950; TAYLOR, 1962).

L'essai réalisé avec des écartements de fils différents montre que, grâce à l'effet combiné de la réduction des intervalles entre fils et de la diminution de la surface d'interception, l'efficacité de ce type de piège est peu sensible aux variations de direction du vent incident, au moins jusqu'à un angle de $43^{\circ}$, la perte d'efficacité ne dépassant pas 6 p. 100. Le piège à fils englués peut donc être utilisé soit monté sur un support à girouette, soit, plus simplement, en associant 2 pièges disposés perpendiculairement l'un par rapport à l'autre. Dans ce cas, la moyenne des captures des 2 pièges associés permettrait d'avoir une bonne estimation des pucerons transportés par des vents de direction quelconque.

Ces résultats, associés à ceux présentés précédemment (LABONNE et al., 1983; 1988a), permettent de mieux cerner les possibilités d'utilisation du piège à fils englués en épidémiologie :

- Il peut servir à évaluer les niveaux de population de pucerons en vol au-dessus d'une culture. Il doit alors soit être monté sur un support à girouette, soit être utilisé par groupes de 2 pièges disposés perpendiculairement l'un à l'autre. Un enregistrement de la vitesse du vent doit être associé à ce type de piégeage.

- Il peut aussi être utilisé pour mesurer la circulation des vecteurs à l'intérieur du couvert végétal constitué par la culture. Il est alors placé verticalement sur le sol, sur la ligne ou dans l'interligne, et il est beaucoup moins affecté par les variations de vitesse et de direction du vent en raison de la rugosité de la culture (LEGG \& BAINBRIDGE, 1978) qui induit une couche limite où les pucerons peuvent la plupart du temps diriger leur vol. 
L'utilisation de couples de pièges perpendiculaires et d'enregistrement de la vitesse du vent n'est alors pas indispensable. Des essais ont montré qu'une relation liait les nombres de pucerons capturés par des pièces à fils englués disposés sur le sol avec la vitesse de progression des épidémies (LABONNE et al., 1988b), ce qui constitue une illustration de l'intérêt de ce type de piégeage.

Recule 13 janvier 1988. Accepté le 11 mars 1988.

\section{RÉFÉRENCES BIBLIOGRAPHIQUES}

Johnson C. G., 1950. The comparison of suction trap, sticky trap and tow-net for the quantitative sampling of small airborne insccts. $\mathrm{Am}$. appl. Biol., $37: 268-285$.

Labonne G., Fauvel G., Leclant F., Quiot J. B., 1983. Intérêt des pièges a fils dans l'étude des populations de pucerons ailés. Agronomie, 3 : 315-326.

Labonne G., Lauriaut F., Quiot J. B., 1988a. Comparaison de 3 types de pièges pour l'échantillonnage des populations de pucerons ailés. Agronomie (à paraître).
Labonne G., Quiot J. B., Quiot L., 1988h. Mise en évidence d'une relation quantitative entre l'évolution épidémique d'un virus nonpersistant et le nombre de ses vecteurs, dans le cas du Papaya ringspot virus type W sur Courgette en Guadeloupe. Agronomic, (à paraître).

Legg B. J., Bainbridge A., 1978. Air movement within a crop : spore dispersal and deposition, p. 103-110. Ih P. R. Scott \& A. Bainbridge, Plant disease epidemiology. Blackwell Scientific Publications.

Taylor L. R., 1962. The absolute efficiency of insect suction traps. Ann. appl. Biol., $\mathbf{5 0}: 405-421$. 